Bull. Korean Math. Soc. 49 (2012), No. 6, pp. 1335-1348

http://dx.doi.org/10.4134/BKMS.2012.49.6.1335

\title{
A HARDY INEQUALITY ON H-TYPE GROUPS
}

\author{
YiNGXIONG XIAO
}

ABstract. We prove a Hardy inequality related to Carnot-Carathéodory distance on H-type groups based on a representation formula on such groups.

\section{Introduction}

The Hardy inequality in $\mathbb{R}^{N}$ states that, for all $u \in C_{0}^{\infty}\left(\mathbb{R}^{N}\right)$ and $N \geq 3$,

$$
\int_{\mathbb{R}^{N}}|\nabla u|^{2} d x \geq \frac{(N-2)^{2}}{4} \int_{\mathbb{R}^{N}} \frac{u^{2}}{|x|^{2}} d x .
$$

Inequality (1.1) has been generalized to Heisenberg group $\mathbb{H}_{n}$ and other nilpotent groups by several authors $([5,6,7,8,10,11,21])$. Especially, the following Hardy inequality holds for $1<p<2 n$ and $u \in C_{0}^{\infty}\left(\mathbb{H}_{n}\right)$ (see [5], Theorem 3.3),

$$
\int_{\mathbb{H}_{n}}\left|\nabla_{H} u\right|^{p} \geq\left(\frac{2 n-p}{p}\right)^{p} \int_{\mathbb{H}_{n}} \frac{|u|^{p}}{d^{p}}
$$

where $d$ is the Korányi-Folland non-isotropic gauge. We note there is another distance, called Carnot-Carathéodory distance on $\mathbb{H}_{n}$ and this distance plays an important role in the study of hypoelliptic heat kernel (see [1, 9, 12, 13, 15, $16,17]$ ). Since $d_{c c}(\xi) \geq d(\xi)$ for all $\xi \in \mathbb{H}_{n}$ (see [18], Lemma 5.1), inequality (1.2) imply the following

$$
\int_{\mathbb{H}_{n}}\left|\nabla_{H} u\right|^{p} \geq\left(\frac{2 n-p}{p}\right)^{p} \int_{\mathbb{H}_{n}} \frac{|u|^{p}}{d_{c c}^{p}} .
$$

However, the constant in (1.3) is not sharp.

The aim of this paper is to give a new proof of Hardy inequalities associated with $d_{c c}$ on H-type groups $G$, a remarkable class of stratified groups of step two introduced by Kaplan [14]. To do so, we prove a representation formula

Received July 4, 2011; Revised November 25, 2011.

2010 Mathematics Subject Classification. Primary 22E25, $26 \mathrm{D} 10$.

Key words and phrases. Hardy inequality, Heisenberg type group, Carnot-Carathéodory distance.

This research is supported by the National Natural Science Foundation of China (No.11101096). 
associated with $d_{c c}$, following the idea of Cohn et al. $([3,4])$ and Zhu $([22])$. Furthermore, the constant we obtain seems sharp (see Remark 4.3).

To state our result, we need some notations (for details, see Section 2). We denote by $\nabla_{G}$ the horizontal gradient on $G$. Let $Q=m+2 n$ be the homogenous dimension of $G$. To this end we have:

Theorem 1.1. Let $1<p<Q-\alpha$. There holds, for all $f \in C_{0}^{\infty}(G)$,

$$
\int_{G} \frac{\left|\nabla_{G} f\right|^{p}}{d_{c c}^{\alpha}} \geq\left(\frac{Q-p-\alpha}{p}\right)^{p} \int_{G} \frac{|f|^{p}}{d_{c c}^{p+\alpha}}
$$

\section{Notations and preliminaries}

Recall that a H-type group $G$ is a Carnot group of step two in which the group law has the form (see [2])

$$
(x, t) \circ\left(x^{\prime}, t^{\prime}\right)=\left(\begin{array}{c}
x_{i}+x_{i}^{\prime}, \quad i=1,2, \ldots, m \\
t_{j}+t_{j}^{\prime}+\frac{1}{2}\left\langle U^{(j)} x, x^{\prime}\right\rangle, \quad j=1,2, \ldots, n
\end{array}\right),
$$

where the matrices $U^{(1)}, U^{(2)}, \ldots, U^{(n)}$ have the following properties:

(1) $U^{(j)}$ is a $m \times m$ skew symmetric and orthogonal matrix for every $j=$ $1,2, \ldots, n$;

(2) $U^{(i)} U^{(j)}+U^{(j)} U^{(i)}=0$ for every $i, j \in\{1,2, \ldots, n\}$ with $i \neq j$.

An easy computation shows that the vector fields in the algebra $\mathfrak{g}$ of $G=$ $\left(\mathbb{R}^{m+n}, \circ\right)$ that agree at the origin with $\frac{\partial}{\partial x_{j}}(j=1, \ldots, m)$ are given by

$$
X_{j}=\frac{\partial}{\partial x_{j}}+\frac{1}{2} \sum_{k=1}^{n}\left(\sum_{i=1}^{m} U_{j, i}^{(k)} x_{i}\right) \frac{\partial}{\partial t_{k}},
$$

and that $\mathfrak{g}$ is spanned by the left-invariant vector fields $X_{1}, \ldots, X_{m}, \frac{\partial}{\partial t_{1}}, \ldots$, $\frac{\partial}{\partial t_{n}}$. The horizontal gradient is the vector given by

$$
\nabla_{G}=\left(X_{1}, \ldots, X_{m}\right)=\nabla_{x}+\frac{1}{2} U^{(1)} x \frac{\partial}{\partial t_{1}}+\cdots+\frac{1}{2} U^{(n)} x \frac{\partial}{\partial t_{n}}
$$

with

$$
x=\left(x_{1}, \ldots, x_{m}\right) \text { and } \nabla_{x}=\left(\frac{\partial}{\partial x_{1}}, \ldots, \frac{\partial}{\partial x_{m}}\right) .
$$

We call a curve $\gamma:[a, b] \rightarrow G$ a horizontal curve connecting two points $\xi, \eta \in G$ if $\gamma(a)=\xi, \gamma(b)=\eta$ and $\dot{\gamma}(s) \in \operatorname{span}\left\{X_{1}, \ldots, X_{m}\right\}$ for all $s$. The Carnot-Carathéodory distance between $\xi, \eta$ is defined as

$$
d_{c c}(\xi, \eta)=\inf _{\gamma} \int_{a}^{b} \sqrt{\langle\dot{\gamma}(s), \dot{\gamma}(s)\rangle} d s
$$

where the infimum is taken over all horizontal curves $\gamma$ connecting $\xi$ and $\eta$. It is known that any two points $\xi, \eta$ on $G$ can be joined by a horizontal curve of 
finite length and then $d_{c c}$ is a metric on $G$. With this norm, we can define the metric ball centered at origin $e$ and with radius $\rho$ by

$$
B_{c c}(e, \rho)=\left\{\eta: d_{c c}(e, \eta)<\rho\right\}
$$

and the unit sphere $\Sigma=\partial B_{c c}(e, 1)$. For simplicity, we write $d_{c c}(\xi)=d_{c c}(e, \xi)$.

For each real number $\lambda>0$, there is a dilation naturally associated with the group structure which is usually denoted as $\delta_{\lambda}(x, t)=\left(\lambda x, \lambda^{2} t\right)$. The Jacobian determinant of $\delta_{\lambda}$ is $\lambda^{Q}$, where $Q=m+2 n$ is the homogenous dimension of $G$. For simplicity, we use the notation $\lambda(x, t)=\left(\lambda x, \lambda^{2} t\right)$. The CarnotCarathéodory distance $d_{c c}$ satisfies

$$
d_{c c}(\lambda(x, t))=\lambda d_{c c}(x, t), \quad \lambda>0 .
$$

Given any $\xi=(x, t) \in G$, set $x^{*}=\frac{z}{d_{c c}(x, t)}, t^{*}=\frac{t}{d_{c c}(x, t)^{2}}$ and $\xi^{*}=\left(x^{*}, t^{*}\right)$ if $d_{c c}(x, t) \neq 0$. The polar coordinates on $G$ associated with $d_{c c}$ is the following (cf. [20]):

$$
\int_{G} f(x, t) d x d t=\int_{0}^{\infty} \int_{\Sigma} f\left(\lambda\left(x^{*}, t^{*}\right)\right) \lambda^{Q-1} d \sigma d \lambda, \quad f \in L^{1}(G) .
$$

Set

$$
\mu(\varphi)=\frac{2 \varphi-\sin 2 \varphi}{2 \sin ^{2} \varphi}:(-\pi, \pi) \rightarrow \mathbb{R}
$$

Then $\mu$ is a diffeomorphism of the interval $(-\pi, \pi)$ onto $\mathbb{R}$ (cf. [1]). We denote by $\mu^{-1}$ the inverse function of $\mu$. The Carnot-Carathéodory distance $d_{c c}$ satisfies (cf. [9])

$$
d_{c c}(x, t)= \begin{cases}\frac{\varphi}{\sin \varphi}|x|, & x \neq 0, t \neq 0 \\ |x|, & t=0 \\ \sqrt{4 \pi|t|}, & x=0\end{cases}
$$

where

$$
\varphi=\mu^{-1}\left(\frac{4|t|}{|x|^{2}}\right), \quad|x|^{2}=\sum_{j=1}^{m} x_{j}^{2} \quad \text { and } \quad|t|^{2}=\sum_{j=1}^{n} t_{j}^{2} .
$$

Let $\mathbb{Z}=\{(x, t) \in G: x=0\}$ be the center of $G$ and $\mathbb{Z}^{\prime}=\{(x, t) \in G: t=0\}$. Since the function $\frac{4|t|}{|x|^{2}}$ is of class $C^{\infty}$ in $G \backslash\left(\mathbb{Z} \cup \mathbb{Z}^{\prime}\right)$, the Carnot-Carathéodory distance $d_{c c}(x, t)$ is of class $C^{\infty}$ in $G \backslash\left(\mathbb{Z} \bigcup \mathbb{Z}^{\prime}\right)$. Therefore (cf. [20])

$$
\left|\nabla_{G} d_{c c}(x, t)\right|=1, \quad(x, t) \in G \backslash\left(\mathbb{Z} \bigcup \mathbb{Z}^{\prime}\right) .
$$

\section{Geodesics in a H-type group}

In this section we shall give a parametrization of $G$ using the geodesics. We refer to [19] the analogous parametrization of Heisenberg groups. Recall that 
the Kokn's sublaplacian on the H-type group $G$ is given by

$$
\begin{aligned}
\Delta_{G} & =\sum_{j=1}^{m} X_{j}^{2}=\sum_{j=1}^{m}\left(\frac{\partial}{\partial x_{j}}+\frac{1}{2} \sum_{k=1}^{n}\left(\sum_{i=1}^{m} U_{j, i}^{(k)} x_{i}\right) \frac{\partial}{\partial t_{k}}\right)^{2} \\
& =\Delta_{x}+\frac{1}{4}|x|^{2} \Delta_{t}+\sum_{k=1}^{n}\left\langle U^{(k)} x, \nabla_{x}\right\rangle \frac{\partial}{\partial t_{k}}
\end{aligned}
$$

where

$$
\Delta_{x}=\sum_{j=1}^{m}\left(\frac{\partial}{\partial x_{j}}\right)^{2}, \Delta_{t}=\sum_{k=1}^{n}\left(\frac{\partial}{\partial t_{k}}\right)^{2} .
$$

The associated Hamiltonian function $H(x, t, \xi, \theta)$ is of the form

$$
\begin{aligned}
H(x, t, \xi, \theta) & =\sum_{j=1}^{m}\left(\xi_{j}+\frac{1}{2} \sum_{k=1}^{n}\left(\sum_{i=1}^{m} U_{j, i}^{(k)} x_{i}\right) \theta_{k}\right)^{2} \\
& =|\xi|^{2}+\frac{1}{4}|x|^{2}|\theta|^{2}+\sum_{k=1}^{n}\left\langle\theta_{k} U^{(k)} x, \xi\right\rangle
\end{aligned}
$$

where

$$
\xi=\left(\xi_{1}, \ldots, \xi_{m}\right)=\left(\frac{\partial}{\partial x_{1}}, \ldots, \frac{\partial}{\partial x_{m}}\right), \theta=\left(\theta_{1}, \ldots, \theta_{n}\right)=\left(\frac{\partial}{\partial t_{1}}, \ldots, \frac{\partial}{\partial t_{n}}\right) .
$$

It has been proved by Eldredge ([9]) that geodesics in $G$ are solutions of the Hamiltonian system associated with Hamiltonian function $H(x, t, \xi, \theta)$. For simplicity, we introduce the notation $M_{\theta}=\sum_{k=1}^{n} \theta_{k} U^{(k)}$. Properties of the matrices $\left\{U^{(1)}, U^{(2)}, \ldots, U^{(n)}\right\}$ give us the following results.

Lemma 3.1. Let $I$ be the identity matric. Put $M_{\theta}=\sum_{k=1}^{n} \theta_{k} U^{(k)}$ and suppose $|\theta| \neq 0$ :

(1) $M_{\theta}^{T}=-M_{\theta}, \quad M_{\theta}^{2}=-|\theta|^{2} \cdot I, \quad M_{\theta}^{-1}=-|\theta|^{-2} M_{\theta}$;

(2) $\exp \left(s M_{\theta}\right)=\cos (s|\theta|) \cdot I+\sin (s|\theta|) \cdot \frac{M_{\theta}}{|\theta|}$.

Proof. (1) By Theorem 2.1,

$$
\begin{aligned}
M_{\theta}^{T} & =\left(\sum_{k=1}^{n} \theta_{k} U^{(k)}\right)^{T}=-\sum_{k=1}^{n} \theta_{k} U^{(k)}=-M_{\theta} \\
M_{\theta}^{2} & =\left(\sum_{k=1}^{n} \theta_{k} U^{(k)}\right)^{2}=\sum_{k=1}^{n} \theta_{k}^{2}\left(U^{(k)}\right)^{2}+\sum_{i<j} \theta_{i} \theta_{j}\left(U^{(i)} U^{(j)}+U^{(j)} U^{(i)}\right) \\
& =-\sum_{k=1}^{n} \theta_{k}^{2} \cdot I=-|\theta|^{2} \cdot I .
\end{aligned}
$$

So $M_{\theta}^{-1}=-|\theta|^{-2} M_{\theta}$. 
(2) Since $M_{\theta}^{2}=-|\theta|^{2} \cdot I$, we have

$$
\begin{aligned}
\exp \left(s M_{\theta}\right) & =\sum_{n=0}^{\infty} \frac{\left(s M_{\theta}\right)^{n}}{n !}=\sum_{n=0}^{\infty} \frac{\left(s M_{\theta}\right)^{2 n+1}}{(2 n+1) !}+\sum_{k=0}^{\infty} \frac{\left(s M_{\theta}\right)^{2 k}}{(2 k) !} \\
& =\frac{M_{\theta}}{|\theta|} \sum_{n=0}^{\infty}(-1)^{n} \frac{(s|\theta|)^{2 n+1}}{(2 n+1) !}+\sum_{k=0}^{\infty}(-1)^{k} \frac{(s|\theta|)^{2 k}}{(2 k) !} \cdot I \\
& =\cos (s|\theta|) \cdot I+\sin (s|\theta|) \cdot \frac{M_{\theta}}{|\theta|}
\end{aligned}
$$

Hamiltonian function $H(x, t, \xi, \theta)$ may be denoted by

$$
\begin{aligned}
H(x, t, \xi, \theta) & =|\xi|^{2}+\frac{1}{4}|x|^{2}|\theta|^{2}+\left\langle M_{\theta} x, \xi\right\rangle \\
& =\left\langle\xi+\frac{1}{2} M_{\theta} x, \xi+\frac{1}{2} M_{\theta} x\right\rangle=\langle\zeta, \zeta\rangle,
\end{aligned}
$$

where $\zeta=\xi+\frac{1}{2} M_{\theta} x$. The corresponding Hamilton's equations for a curve $(x(s), t(s), \xi(s), \theta(s))$ take the form

$$
\left\{\begin{array}{l}
\dot{x}(s)=\frac{\partial H}{\partial \xi}=2 \zeta(s) \\
\dot{\xi}(s)=-\frac{\partial H}{\partial x}=M_{\theta} \zeta(s) \\
\dot{t}_{j}(s)=\frac{\partial H}{\partial \theta_{k}}=\left\langle\zeta(s), U^{(j)} x(s)\right\rangle, \quad j=1, \ldots, n, \\
\dot{\theta}(s)=-\frac{\partial H}{\partial t}=0, \text { i.e., } \theta(s)=\theta(0),
\end{array}\right.
$$

Assume $(x(0), t(0))=(0,0)$. We solve the Hamiltonian system explicitly bellow. By $(3.1), \theta$ is constant, and we take it to be the free parameter. The equations (3.1) imply that:

$$
\dot{\zeta}(s)=\dot{\xi}(s)+\frac{1}{2} M_{\theta} \dot{x}(s)=2 M_{\theta} \zeta(s)
$$

Therefore,

$$
\zeta(s)=e^{2 s M_{\theta}} \zeta(0)
$$

and by Lemma 3.1,

$$
\begin{aligned}
x(s) & =2 \int_{0}^{s} \zeta(r) d r=M_{\theta}^{-1}(\zeta(s)-\zeta(0))=M_{\theta}^{-1}\left(I-e^{-2 s M_{\theta}}\right) \zeta(s) \\
& =M_{\theta}^{-1}\left(e^{2 s M_{\theta}}-I\right) \zeta(0)=(\cos (2 s|\theta|)-1) \cdot M_{\theta}^{-1} \zeta(0)+\sin (2 s|\theta|) \cdot \frac{\zeta(0)}{|\theta|} \\
& =(1-\cos (2 s|\theta|)) \cdot \frac{M_{\theta} \zeta(0)}{|\theta|^{2}}+\sin (2 s|\theta|) \cdot \frac{\zeta(0)}{|\theta|}
\end{aligned}
$$


We now describe the $t$-components of a geodesic curve. By (3.1) and (3.2)

$$
\begin{aligned}
\dot{t}_{k}(s) & =\left\langle\zeta(s), U^{(k)} x(s)\right\rangle \\
& =\left\langle\frac{1}{2} \dot{x}(s), U^{(k)} x(s)\right\rangle \\
& =\left\langle\frac{1}{2} \dot{x}(s), \frac{1}{2} U^{(k)} M_{\theta}^{-1}\left(I-e^{-2 s M_{\theta}}\right) \zeta(s)\right\rangle \\
& =\left\langle\frac{1}{2} \dot{x}(s), \frac{1}{2} U^{(k)} M_{\theta}^{-1}\left(I-e^{-2 s M_{\theta}}\right) \dot{x}(s)\right\rangle .
\end{aligned}
$$

A simply calculation gives us

$$
\begin{aligned}
U^{(k)} M_{\theta}^{-1}\left(e^{2 s M_{\theta}}-I\right) & =U^{(k)} M_{\theta}^{-1}-U^{(k)} M_{\theta}^{-1} e^{-2 s M_{\theta}} \\
& =U^{(k)} M_{\theta}^{-1}-U^{(k)} M_{\theta}^{-1}\left(\cos 2 s|\theta|-\frac{M_{\theta}}{|\theta|} \sin 2 s|\theta|\right) \\
& =(1-\cos 2 s|\theta|) U^{(k)} M_{\theta}^{-1}-\frac{\sin 2 s|\theta|}{|\theta|} U^{(k)}
\end{aligned}
$$

By Lemma 3.1,

$$
M^{-1}=-|\theta|^{-2} M_{\theta}=-|\theta|^{-2} \sum_{i=1}^{n} \theta_{i} U^{(i)}
$$

and we obtain, by (3.4),

$$
\begin{aligned}
& U^{(k)} M_{\theta}^{-1}\left(e^{2 s M_{\theta}}-I\right) \\
= & (1-\cos 2 s|\theta|) U^{(k)} M_{\theta}^{-1}-\frac{\sin 2 s|\theta|}{|\theta|} U^{(k)} \\
= & -\frac{(1-\cos 2 s|\theta|)}{|\theta|^{2}} U^{(k)} \sum_{i=1}^{n} \theta_{i} U^{(i)}-\frac{\sin 2 s|\theta|}{|\theta|} U^{(k)} \\
= & \frac{1-\cos 2 s|\theta|}{|\theta|^{2}} \theta_{k} \cdot I-\frac{1-\cos 2 s|\theta|}{|\theta|^{2}} \sum_{i \neq k} \theta_{i} U^{(k)} U^{(i)}-\frac{\sin 2 s|\theta|}{|\theta|} U^{(k)} .
\end{aligned}
$$

Properties of the matrices $\left\{U^{(1)}, U^{(2)}, \ldots, U^{(n)}\right\}$ imply $U^{(k)} U^{(i)}(i \neq k)$ is also a $m \times m$ skew symmetric and orthogonal matrix. Therefore we get, for $i \neq k$,

$$
\left\langle U^{(k)} U^{(i)} \dot{x}(s), \dot{x}(s)\right\rangle=0
$$

and also

$$
\left\langle U^{(k)} \dot{x}(s), \dot{x}(s)\right\rangle=0, \quad k=1, \ldots, n .
$$


Thus, by (3.3) and (3.5),

$$
\begin{aligned}
\dot{t}_{k}(s) & =\frac{1-\cos 2 s|\theta|}{4|\theta|^{2}} \theta_{k}\langle\dot{x}(s), \dot{x}(s)\rangle \\
& =\frac{1-\cos 2 s|\theta|}{4|\theta|^{2}} \theta_{k}\left\langle e^{2 s M_{\theta}} \zeta(0), e^{2 s M_{\theta}} \zeta(0)\right\rangle \\
& =\frac{1-\cos 2 s|\theta|}{4|\theta|^{2}} \theta_{k}\langle\zeta(0), \zeta(0)\rangle .
\end{aligned}
$$

Integrating equations (3.6) we obtain, for $k=1, \ldots, n$,

$$
t_{k}(s)=\frac{\theta_{k}}{4|\theta|^{2}}\left(s-\frac{\sin 2 s|\theta|}{2|\theta|}\right)\langle\zeta(0), \zeta(0)\rangle
$$

Taking the initial date $(x(0), t(0))=(0,0)$ and $(\zeta(0), \theta(0))=(A, \phi / 2)=$ $\left(A_{1}, \ldots, A_{m}, \phi_{1} / 2, \ldots, \phi_{n} / 2\right)$, we find the solutions

$$
\left\{\begin{array}{l}
x(s)=(1-\cos (s|\phi|)) \cdot \frac{M_{\phi} A}{|\phi|^{2}}+\sin (s|\phi|) \cdot \frac{A}{|\phi|}, \\
t(s)=\frac{\phi}{2|\phi|} \cdot \frac{|\phi| s-\sin s|\phi|}{|\phi|^{2}}\langle A, A\rangle,
\end{array}\right.
$$

where $M_{\phi}=\sum_{k=1}^{n} \phi_{k} U^{(k)}$. Letting $|\phi| \rightarrow 0+$, we get the Euclidean geodesics

$$
(x(s), t(s))=(\zeta(0) s, 0)
$$

and hence the correct normalization is $\langle\zeta(0), \zeta(0)\rangle=\sum_{i=1}^{m} A_{i}^{2}=1$.

Set

$$
\Omega=\left\{(A, \phi, \rho) \in \mathbb{R}^{m} \times \mathbb{R}^{n} \times \mathbb{R}:|A|^{2}=\sum_{j=1}^{m} A_{j}^{2}=1,0 \leq|\phi| \rho \leq 2 \pi, \rho \geq 0\right\} .
$$

and define $\Phi: \Omega \rightarrow G$ by $\Phi(A, \phi, \rho)=(x(A, \phi, \rho), t(A, \phi, \rho))$, where

$$
\left\{\begin{array}{l}
x(A, \phi, \rho)=(1-\cos (|\phi| \rho)) \cdot \frac{M_{\phi} A}{|\phi|^{2}}+\sin (|\phi| \rho) \cdot \frac{A}{|\phi|} \\
t(A, \phi, \rho)=\frac{\phi}{2|\phi|} \cdot \frac{|\phi| \rho-\sin (|\phi| \rho)}{|\phi|^{2}}
\end{array}\right.
$$

and $M_{\phi}=\sum_{k=1}^{n} \phi_{k} U^{(k)}$. We note the range of $\Phi$ is $G$ and the center $\mathbb{Z}$ is just the set of points $\Phi(A, \phi, \rho)$ with $|\phi| \rho=2 \pi$ and the set $\mathbb{Z}^{\prime}$ is just the set of points $\Phi(A, \phi, \rho)$ with $|\phi|=0$. Furthermore, if one fixes $\rho>0$, equations (3.7) parameterize $\partial B_{c c}(e, \rho)$.

By (3.7),

$$
|x|^{2}=(1-\cos (|\phi| \rho))^{2} \cdot \frac{|\phi|^{2}}{|\phi|^{4}}+\sin ^{2}(|\phi| \rho) \cdot \frac{1}{|\phi|^{2}}=\frac{2(1-\cos (|\phi| \rho))}{|\phi|^{2}}
$$

and

$$
\mu(\varphi)=\frac{4|t|}{|x|^{2}}=\frac{|\phi| \rho-\sin (|\phi| \rho)}{1-\cos (|\phi| \rho)}=\frac{|\phi| \rho-\sin (|\phi| \rho)}{2 \sin ^{2}\left(\frac{|\phi| \rho}{2}\right)}=\mu\left(\frac{|\phi| \rho}{2}\right)
$$


Therefore,

$$
\varphi=\frac{|\phi| \rho}{2}, \quad|\phi| \rho \in[0,2 \pi)
$$

since $\mu$ is a diffeomorphism of the interval $(-\pi, \pi)$ onto $\mathbb{R}$.

\section{The proof}

For any $\delta>0$, set $\mathbb{Z}_{\delta}=(-\delta, \delta)^{m} \times \mathbb{R}^{n}, \mathbb{Z}_{\delta}^{\prime}=\mathbb{R}^{m} \times(-\delta, \delta)^{n}$ and $\Sigma_{\delta}=$ $\Sigma \backslash\left(\mathbb{Z}_{\delta} \cup \mathbb{Z}_{\delta}^{\prime}\right)$. Note that $\mathbb{Z}_{\delta}$ and $\mathbb{Z}_{\delta}^{\prime}$ are open sets that contain $\mathbb{Z}$ and $\mathbb{Z}^{\prime}$, respectively. Now we prove the following representation formula. The proof is similar to that of $[3,4]$ and $[22]$.

Lemma 4.1. Let $R_{2}>R_{1}>0$ and $f \in C(G) \cap C^{1}\left(G \backslash\left(\mathbb{Z} \cup \mathbb{Z}^{\prime}\right)\right)$. There holds

$$
\int_{\Sigma_{\delta}} f\left(R_{2} \xi^{*}\right) d \sigma-\int_{\Sigma_{\delta}} f\left(R_{1} \xi^{*}\right) d \sigma=\int_{R_{1}}^{R_{2}} \int_{\Sigma_{\delta}}\left\langle\nabla_{G} f, \nabla_{G} d_{c c}\right\rangle d \sigma d \rho .
$$

Proof. We have, for $\xi^{*} \in \Sigma_{\delta}$,

$$
\begin{aligned}
f\left(R_{2} \xi^{*}\right) d-f\left(R_{1} \xi^{*}\right) & =\int_{R_{1}}^{R_{2}} \frac{d}{d \rho} f\left(\rho \xi^{*}\right) d \rho \\
& =\int_{R_{1}}^{R_{2}}\left(\left\langle\nabla_{x} f(\xi), \frac{\partial x}{\partial \rho}\right\rangle+\left\langle\nabla_{t} f(\xi), \frac{\partial t}{\partial \rho}\right\rangle\right) d \rho .
\end{aligned}
$$

Therefore,

$$
\begin{aligned}
& \int_{\Sigma_{\delta}} f\left(R_{2} \xi^{*}\right) d \sigma-\int_{\Sigma_{\delta}} f\left(R_{1} \xi^{*}\right) d \sigma \\
= & \int_{\Sigma_{\delta}} \int_{R_{1}}^{R_{2}} \frac{d}{d \rho} f\left(\rho \xi^{*}\right) d \rho d \sigma \\
= & \int_{\Sigma_{\delta}} \int_{R_{1}}^{R_{2}}\left(\left\langle\nabla_{x} f(\xi), \frac{\partial x}{\partial \rho}\right\rangle+\left\langle\nabla_{t} f(\xi), \frac{\partial t}{\partial \rho}\right\rangle\right) d \rho d \sigma
\end{aligned}
$$

where $\xi=(x, t)=\rho \xi^{*}$. By (3.7) and (2.2),

$$
\begin{aligned}
& \left\langle\nabla_{x} f(\xi), \frac{\partial x}{\partial \rho}\right\rangle+\left\langle\nabla_{t} f(\xi), \frac{\partial t}{\partial \rho}\right\rangle \\
= & \left\langle\nabla_{x} f(\xi), \sin (|\phi| \rho) \cdot \frac{M_{\phi} A}{|\phi|}+\cos (|\phi| \rho) A\right\rangle+\left\langle\nabla_{t} f(\xi), \frac{\phi}{2|\phi|} \cdot \frac{1-\cos (|\phi| \rho)}{|\phi|}\right\rangle \\
= & \left\langle\nabla_{G} f(\xi), \sin (|\phi| \rho) \cdot \frac{M_{\phi} A}{|\phi|}+\cos (|\phi| \rho) A\right\rangle \\
& +\frac{1}{2} \sum_{k=1}^{n}\left(\frac{\phi_{k}}{|\phi|} \cdot \frac{1-\cos (|\phi| \rho)}{|\phi|}-\left\langle U^{(k)} x, \sin (|\phi| \rho) \cdot \frac{M_{\phi} A}{|\phi|}+\cos (|\phi| \rho) A\right\rangle\right) \frac{\partial f}{\partial t_{k}} .
\end{aligned}
$$


Again using (3.7), we have, since $U^{(k)}$ and $U^{(k)} U^{(j)}(j \neq k)$ are $m \times m$ skew symmetric matrixes,

$$
\begin{aligned}
& \left\langle U^{(k)} x, \sin (|\phi| \rho) \cdot \frac{M_{\phi} A}{|\phi|}+\cos (|\phi| \rho) A\right\rangle \\
= & \left\langle(1-\cos (|\phi| \rho)) \frac{U^{(k)} M_{\phi} A}{|\phi|^{2}}+\sin (|\phi| \rho) \frac{U^{(k)} A}{|\phi|}, \sin (|\phi| \rho) \frac{M_{\phi} A}{|\phi|}+\cos (|\phi| \rho) A\right\rangle \\
= & \frac{(1-\cos (|\phi| \rho)) \sin (|\phi| \rho)}{|\phi|^{3}}\left\langle U^{(k)} M_{\phi} A, M_{\phi} A\right\rangle+\frac{(\cos (|\phi| \rho) \sin (|\phi| \rho)}{|\phi|}\left\langle U^{(k)} A, A\right\rangle \\
& +\left(\frac{(1-\cos (|\phi| \rho)) \cos (|\phi| \rho)}{|\phi|^{2}}-\frac{\left(\sin ^{2}(|\phi| \rho)\right.}{|\phi|^{2}}\right)\left\langle U^{(k)} M_{\phi} A, A\right\rangle \\
= & \frac{\cos (|\phi| \rho)-1}{|\phi|^{2}}\left\langle U^{(k)} M_{\phi} A, A\right\rangle \\
= & \frac{1-\cos (|\phi| \rho)}{|\phi|^{2}} \phi_{k}+\frac{\cos (|\phi| \rho)-1}{|\phi|^{2}} \sum_{j \neq k}\left\langle U^{(k)} U^{(j)} A, A\right\rangle \phi_{j} \\
= & \frac{1-\cos (|\phi| \rho)}{|\phi|^{2}} \phi_{k} .
\end{aligned}
$$

Therefore, we have, by (4.2) and (4.3),

$$
\begin{aligned}
& \int_{\Sigma_{\delta}} f\left(R_{2} \xi^{*}\right) d \sigma-\int_{\Sigma_{\delta}} f\left(R_{1} \xi^{*}\right) d \sigma \\
= & \int_{\Sigma_{\delta}} \int_{R_{1}}^{R_{2}}\left\langle\nabla_{G} f(\xi), \sin (|\phi| \rho) \cdot \frac{M_{\phi} A}{|\phi|}+\cos (|\phi| \rho) A\right\rangle d \rho d \sigma .
\end{aligned}
$$

To finish the proof, it is enough to show

$$
\nabla_{G} d_{c c}(x, t)=\sin (|\phi| \rho) \frac{M_{\phi} A}{|\phi|}+\cos (|\phi| \rho) A, \quad(x, t) \in G \backslash\left(\mathbb{Z} \bigcup \mathbb{Z}^{\prime}\right) .
$$

This is just the following Lemma 4.2. The proof of Lemma 4.1 is now completed.

Lemma 4.2. There holds, for $(x, t) \in G \backslash\left(\mathbb{Z} \bigcup \mathbb{Z}^{\prime}\right)$,

$$
\nabla_{G} d_{c c}(x, t)=\sin (|\phi| \rho) \frac{M_{\phi} A}{|\phi|}+\cos (|\phi| \rho) A .
$$

Proof. Recall that if $(x, t) \in G \backslash\left(\mathbb{Z} \cup \mathbb{Z}^{\prime}\right)$, then

$$
d_{c c}(x, t)=\frac{\varphi}{\sin \varphi}|x|, \quad \varphi=\mu^{-1}\left(\frac{4|t|}{|x|^{2}}\right) .
$$

Though a simple calculation, we have

$$
\mu^{\prime}(\varphi)=\frac{2 \sin \varphi-2 \varphi \cos \varphi}{\sin ^{3} \varphi} ;
$$




$$
\begin{aligned}
\nabla_{x} \varphi & =\frac{1}{\mu^{\prime}(\varphi)} \cdot \frac{-8|t| x}{|x|^{4}} \\
& =-\frac{4|t| x}{|x|^{4}} \cdot \frac{\sin ^{3} \varphi}{\sin \varphi-\varphi \cos \varphi} ; \\
\nabla_{t} \varphi & =\frac{1}{\mu^{\prime}(\varphi)} \cdot \frac{-4 t}{|x|^{2}|t|} \\
& =-\frac{2 t}{|x|^{2}|t|} \cdot \frac{\sin ^{3} \varphi}{\sin \varphi-\varphi \cos \varphi} ; \\
\nabla_{x} d_{c c}(x, t) & =\nabla_{x}\left(\frac{\varphi}{\sin \varphi}|x|\right) \\
& =\frac{\varphi \cos \varphi-\sin \varphi}{\sin ^{2} \varphi}|x| \nabla_{x} \varphi+\frac{\varphi}{\sin \varphi} \cdot \frac{x}{|x|} \\
& =-\frac{4 \sin \varphi|t| x}{|x|^{3}}+\frac{\varphi}{\sin \varphi} \cdot \frac{x}{|x|} \\
& =-\mu(\varphi) \sin \varphi \cdot \frac{x}{|x|}+\frac{\varphi}{\sin \varphi \cdot \frac{x}{|x|}} \\
& =\cos \varphi \cdot \frac{x}{|x|} ; \\
\nabla_{t} d_{c c}(x, t)=\nabla_{t}\left(\frac{\varphi}{\sin \varphi}\right. & |x|)=\frac{\varphi \cos \varphi-\sin \varphi}{\sin ^{2} \varphi}|x| \nabla_{t} \varphi=\frac{2 \sin \varphi}{|x|} \cdot \frac{t}{|t|} .
\end{aligned}
$$

Therefore, we obtain, by (2.2) and (3.7),

$$
\begin{aligned}
\nabla_{G} d_{c c}(x, t) & =\nabla_{x} d_{c c}(x, t)+\frac{1}{2} \sum_{k=1}^{n} U^{(k)} x \frac{\partial d_{c c}(x, t)}{\partial t_{k}} \\
& =\cos \varphi \cdot \frac{x}{|x|}+\frac{\sin \varphi}{|x|} \cdot\left(\sum_{k=1}^{n} \frac{t_{k}}{|t|} \cdot U^{(k)}\right) x \\
& =\cos \varphi \cdot \frac{x}{|x|}+\frac{\sin \varphi}{|x|} \cdot\left(\sum_{k=1}^{n} \frac{\phi_{k}}{|\phi|} \cdot U^{(k)}\right) x \\
& =\cos \varphi \cdot \frac{x}{|x|}+\sin \varphi \cdot \frac{M_{\phi} x}{|x||\phi|} .
\end{aligned}
$$

On the other hand, by (3.7),

$$
|x|=\sqrt{(1-\cos (|\phi| \rho))^{2} \cdot \frac{|\phi|^{2}}{|\phi|^{4}}+\frac{\sin ^{2}(|\phi| \rho)}{|\phi|^{2}}}=\sqrt{\frac{2(1-\cos (|\phi| \rho))}{|\phi|^{2}}}=\frac{2 \sin \left(\frac{|\phi| \rho}{2}\right)}{|\phi|}
$$

and

$$
\frac{x}{|x|}=\frac{(1-\cos (|\phi| \rho)) M_{\phi} A}{|\phi| \cdot 2 \sin \left(\frac{|\phi| \rho}{2}\right)}+\frac{\sin (|\phi| \rho)}{2 \sin \left(\frac{|\phi| \rho}{2}\right)} \cdot A=\frac{\sin \left(\frac{|\phi| \rho}{2}\right)}{|\phi|} M_{\phi} A+\cos \left(\frac{|\phi| \rho}{2}\right) A .
$$


Thus,

$$
\begin{aligned}
& \nabla_{G} d_{c c}(x, t) \\
= & \cos \varphi \cdot \frac{x}{|x|}+\sin \varphi \cdot \frac{M_{\phi} x}{|x||\phi|} \\
= & \cos \varphi \cdot\left(\frac{\sin \left(\frac{|\phi| \rho}{2}\right)}{|\phi|} M_{\phi} A+\cos \left(\frac{|\phi| \rho}{2}\right) A\right) \\
& +\frac{\sin \varphi}{|\varphi|} \cdot\left(\frac{\sin \left(\frac{|\phi| \rho}{2}\right)}{|\phi|} M_{\phi}^{2} A+\cos \left(\frac{|\phi| \rho}{2}\right) M_{\phi} A\right) \\
= & \cos \varphi \cdot\left(\frac{\sin \left(\frac{|\phi| \rho}{2}\right)}{|\phi|} M_{\phi} A+\cos \left(\frac{|\phi| \rho}{2}\right) A\right) \\
& +\frac{\sin \varphi}{|\varphi|} \cdot\left(-\frac{\sin \left(\frac{|\phi| \rho}{2}\right)}{|\phi|}|\phi|^{2} A+\cos \left(\frac{|\phi| \rho}{2}\right) M_{\phi} A\right) \quad \text { (by Lemma 3.1) } \\
= & \cos \left(\varphi+\frac{|\phi| \rho}{2}\right) A+\frac{\sin \left(\varphi+\frac{|\phi| \rho}{2}\right)}{|\phi|} M_{\phi} A \\
= & \left.\cos (|\phi| \rho) A+\frac{\sin (|\phi| \rho)}{|\phi|} M_{\phi} A \text {. (by }(3.8)\right)
\end{aligned}
$$

This completes the proof of Lemma 4.2.

Proof of Theorem 1.1. Let $\epsilon>0$ and set $f_{\epsilon}:=\left(|f|^{2}+\epsilon^{2}\right)^{p / 2}-\epsilon^{p} . f_{\epsilon}$ has the same support as $f$ and hence $f_{\epsilon} \in C_{0}^{\infty}(G)$. Since $d_{c c}(x, t) \in C(G) \cap$ $C^{1}\left(G \backslash\left(\mathbb{Z} \bigcup \mathbb{Z}^{\prime}\right)\right)$, we can replace $f$ with $f_{\epsilon} d_{c c}^{Q-p-\alpha}$ in Lemma 4.1 and obtain, for $R_{2}>R_{1}>0$ and any $\delta>0$,

$$
\begin{aligned}
& (Q-p-\alpha) \int_{R_{1}}^{R_{2}} \int_{\Sigma_{\delta}} f_{\epsilon} d_{c c}^{Q-p-\alpha-1} d \sigma d \rho \\
& -R_{2}^{Q-p-\alpha} \int_{\Sigma_{\delta}} f_{\epsilon}\left(R_{2} u^{*}\right) d \sigma+R_{1}^{Q-p-\alpha} \int_{\Sigma_{\delta}} f_{\epsilon}\left(R_{1} u^{*}\right) d \sigma \\
= & -p \int_{R_{1}}^{R_{2}} \int_{\Sigma_{\delta}}\left(|f|^{2}+\epsilon^{2}\right)^{(p-2) / 2} f\left\langle\nabla_{G} f, \nabla_{G} d_{c c}\right\rangle d_{c c}^{Q-p-\alpha} d \sigma d \rho \\
\leq & p \int_{R_{1}}^{R_{2}} \int_{\Sigma_{\delta}}\left(|f|^{2}+\epsilon^{2}\right)^{(p-2) / 2}|f| \cdot\left|\nabla_{G} f\right| d_{c c}^{Q-p-\alpha} d \sigma d \rho \\
\leq & p \int_{R_{1}}^{R_{2}} \int_{\Sigma_{\delta}}\left(|f|^{2}+\epsilon^{2}\right)^{(p-1) / 2}\left|\nabla_{G} f\right| d_{c c}^{Q-p-\alpha} d \sigma d \rho .
\end{aligned}
$$

Letting $\delta \rightarrow 0$ yields

$$
(Q-p-\alpha) \int_{R_{1}}^{R_{2}} \int_{\Sigma} f_{\epsilon} d_{c c}^{Q-p-\alpha-1} d \sigma d \rho
$$




$$
\begin{aligned}
& -R_{2}^{Q-p-\alpha} \int_{\Sigma} f_{\epsilon}\left(R_{2} u^{*}\right) d \sigma+R_{1}^{Q-p-\alpha} \int_{\Sigma} f_{\epsilon}\left(R_{1} u^{*}\right) d \sigma \\
\leq & p \int_{R_{1}}^{R_{2}} \int_{\Sigma}\left(|f|^{2}+\epsilon^{2}\right)^{(p-1) / 2}\left|\nabla_{G} f\right| d_{c c}^{Q-p-\alpha} d \sigma d \rho .
\end{aligned}
$$

Letting $R_{2} \rightarrow+\infty$ and $R_{1} \rightarrow 0+$, we obtain,

$$
\begin{aligned}
& (Q-p-\alpha) \int_{0}^{\infty} \int_{\Sigma} f_{\epsilon} d_{c c}^{Q-p-\alpha-1} d \sigma d \rho \\
\leq & p \int_{0}^{\infty} \int_{\Sigma}\left(|f|^{2}+\epsilon^{2}\right)^{(p-1) / 2}\left|\nabla_{G} f\right| d_{c c}^{Q-p-\alpha} d \sigma d \rho \\
= & p \int_{G} \frac{\left(|f|^{2}+\epsilon^{2}\right)^{(p-1) / 2} \cdot\left|\nabla_{G} f\right|}{d_{c c}^{p+\alpha-1}} .
\end{aligned}
$$

By dominated convergence, letting $\epsilon \rightarrow 0+$ yields

$$
(Q-p-\alpha) \int_{G} \frac{|f|^{p}}{d_{c c}^{p+\alpha}} \leq p \int_{G} \frac{|f|^{p-1} \cdot\left|\nabla_{G} f\right|}{d_{c c}^{p+\alpha-1}} .
$$

By Hölder's inequality,

$$
(Q-p-\alpha) \int_{G} \frac{|f|^{p}}{d_{c c}^{p+\alpha}} \leq p\left(\int_{G} \frac{|f|^{p}}{d_{c c}^{p+\alpha}}\right)^{\frac{p-1}{p}}\left(\int_{G} \frac{\left|\nabla_{G} f\right|^{p}}{d_{c c}^{\alpha}}\right)^{\frac{1}{p}} .
$$

Canceling and raising both sides to the power $p$, we obtain (1.4).

Remark 4.3. We fail to prove that the constant is sharp in Theorem 1.1. The reason is that we do not know whether the radial function $F_{\epsilon}\left(d_{c c}\right)=$ $g_{\epsilon}\left(d_{c c}\right) \phi\left(d_{c c}\right)$ can be approximated by the functions in $C_{0}^{\infty}(G)$ since $d_{c c}$ is not differentiable in $\mathbb{Z} \cup \mathbb{Z}^{\prime}$, where

$$
g_{\varepsilon}\left(d_{c c}\right)= \begin{cases}\epsilon^{-(Q-p-\alpha) / p}, & d_{c c} \leq \epsilon \\ d_{c c}^{-(Q-p-\alpha) / p}, & d_{c c}>\epsilon\end{cases}
$$

and $\phi(\cdot) \in C_{0}^{\infty}([0,+\infty))$ is a cutoff function satisfying $\phi(t)=1$ if $0 \leq t \leq 1$ and $\phi(t)=0$ if $t \geq 2$. Since $d_{c c}$ is a Lipschitz function, so does $F_{\epsilon}$. Therefore, for all $\varphi \in C_{0}^{\infty}(G)$ (see [20], pages 351-352),

$$
\begin{aligned}
& \int_{G} X_{j} F_{\epsilon}\left(d_{c c}(x, t)\right) \varphi(x, t) d x d t \\
= & -\int_{G} F_{\epsilon}\left(d_{c c}(x, t)\right) X_{j} \varphi(x, t) d x d t, \quad j=1,2, \ldots, m,
\end{aligned}
$$

which means the distribution derivatives $\left\{X_{j} F_{\epsilon}: j=1,2, \ldots, m\right\}$ exist. It is easy to check that $F_{\epsilon}$ is a differentiable function on $\{(x, t) \in G: x \neq 0, z \neq$ $\left.0, d_{c c}(x, t) \neq \epsilon\right\}$. So the distribution derivatives

$$
\begin{aligned}
& X_{j} F_{\epsilon}\left(d_{c c}(x, t)\right)=F_{\epsilon}^{\prime} \cdot X_{j} d_{c c}(x, t), \\
& (x, t) \in\left\{(x, t) \in G: x \neq 0, z \neq 0, d_{c c}(x, t) \neq \epsilon\right\},
\end{aligned}
$$


for $j=1,2, \ldots, m$. We compute

$$
\begin{aligned}
\int_{G} \frac{\left|\nabla_{G} F_{\varepsilon}\right|^{p}}{d_{c c}^{\alpha}} & =|\Sigma| \int_{0}^{2}\left|F_{\epsilon}^{\prime}(\rho)\right|^{p} \rho^{Q-1-\alpha} d \rho \\
& =|\Sigma| \int_{\epsilon}^{1}\left|g_{\epsilon}^{\prime}(\rho)\right|^{p} \rho^{Q-1-\alpha} d \rho+|\Sigma| \int_{1}^{2}\left|F_{\epsilon}^{\prime}(\rho)\right|^{p} \rho^{Q-1-\alpha} d \rho \\
& =-\left(\frac{Q-p-\alpha}{p}\right)^{p}|\Sigma| \ln \epsilon+|\Sigma| \int_{1}^{2}\left|F_{\epsilon}^{\prime}(\rho)\right|^{p} \rho^{Q-1-\alpha} d \rho
\end{aligned}
$$

and

$$
\begin{aligned}
\int_{G} \frac{\left|F_{\varepsilon}\right|^{p}}{d_{c c}^{p+\alpha}}= & |\Sigma| \int_{0}^{2}\left|F_{\epsilon}(\rho)\right|^{p} \rho^{Q-1-p-\alpha} d \rho \\
= & |\Sigma| \int_{0}^{\epsilon}\left|g_{\epsilon}(\rho)\right|^{p} \rho^{Q-1-p-\alpha} d \rho+|\Sigma| \int_{\epsilon}^{1}\left|g_{\epsilon}(\rho)\right|^{p} \rho^{Q-1-p-\alpha} d \rho \\
& +|\Sigma| \int_{1}^{2}\left|F_{\epsilon}(\rho)\right|^{p} \rho^{Q-1-p-\alpha} d \rho \\
= & \frac{|\Sigma|}{Q-p-\alpha}-|\Sigma| \ln \epsilon+|\Sigma| \int_{1}^{2}\left|F_{\epsilon}(\rho)\right|^{p} \rho^{Q-1-p-\alpha} d \rho,
\end{aligned}
$$

where $|\Sigma|=\int_{\Sigma} d \sigma$. Since $\int_{1}^{2}\left|F_{\epsilon}^{\prime}(\rho)\right|^{p} \rho^{Q-1-\alpha} d \rho$ and $\int_{1}^{2}\left|F_{\epsilon}(\rho)\right|^{p} \rho^{Q-1-p-\alpha} d \rho$ are independent of $\epsilon$, we have

$$
\lim _{\varepsilon \rightarrow 0} \frac{\int_{G} \frac{\left|\nabla_{G} F_{\varepsilon}\right|^{p}}{d_{c c}^{\alpha}}}{\int_{G} \frac{\left|F_{\varepsilon}\right|^{p}}{d_{c c}^{p+\alpha}}}=\left(\frac{Q-p-\alpha}{p}\right)^{p} .
$$

If the function $F_{\varepsilon}\left(d_{c c}\right) \in \overline{C_{0}^{\infty}(G)}$, then the constant in Theorem 1.1 is sharp.

Acknowledgements. The authors thanks the referee for his/her careful reading and very useful comments which improved the final version of this paper.

\section{References}

[1] R. Beals, B. Gaveau, and P. C. Greiner, Hamilton-Jacobi theory and the heat kernel on Heisenberg groups, J. Math. Pures Appl. (9) 79 (2000), no. 7, 633-689.

[2] A. Bonfiglioli and F. Uguzzoni, Nonlinear Liouville theorems for some critical problems on H-type groups, J. Funct. Anal. 207 (2004), no. 1, 161-215.

[3] W. Cohn and G. Lu, Best constants for Moser-Trudinger inequalities on the Heisenberg group, Indiana Univ. Math. J. 50 (2001), no. 4, 1567-1591.

[4] , Best constants for Moser-Trudinger inequalities, fundamental solutions and one-parameter representation formulas on groups of Heisenberg type, Acta Math. Sin. (Engl. Ser.) 18 (2002), no. 2, 375-390.

[5] L. D'Ambrosio, Some Hardy inequalities on the Heisenberg group, Differ. Equ. 40 (2004), no. $4,552-564$.

[6] - Hardy inequalities related to Grushin type operators, Proc. Amer. Math. Soc. 132 (2004), no. 3, 725-734.

[7] _ Hardy-type inequalities related to degenerate elliptic differential operators, Ann. Sc. Norm. Super. Pisa Cl. Sci. (5) 4 (2005), no. 3, 451-486. 
[8] D. Danielli, N. Garofalo, and N. C. Phuc, Hardy-Sobolev type inequalities with sharp constants in Carnot-Carathéodory spaces, Potential Anal. 34 (2011), no. 3, 223-242.

[9] N. Eldredge, Precise estimates for the subelliptic heat kernel on H-type groups, J. Math. Pures Appl. (9) 92 (2009), no. 1, 52-85.

[10] N. Garofalo and E. Lanconelli, Frequency functions on the Heisenberg group, the uncertainty principle and unique continuation, Ann. Inst. Fourier (Grenoble) 40 (1990), no. $2,313-356$.

[11] J. A. Goldsteinand and Q. S. Zhang, On a degenerate heat equation with a singular potential, J. Funct. Anal. 186 (2001), no. 2, 342-359.

[12] J.-Q. Hu and H.-Q. Li, Gradient estimates for the heat semigroup on H-type groups, Potential Anal. 33 (2010), no. 4, 355-386.

[13] H. Hueber and D. Müller, Asymptotics for some Green kernels on the Heisenberg group and the Martin boundary, Math. Ann. 283 (1989), no. 1, 97-119.

[14] A. Kaplan, Fundamental solution for a class of hypoelliptic PDE generated by composition of quadratic forms, Trans. Amer. Math. Soc. 258 (1980), no. 1, 147-153.

[15] H.-Q. Li, Estimation optimale du gradient du semi-groupe de la chaleur sur le groupe de Heisenberg, J. Funct. Anal. 236 (2006), no. 2, 369-394.

[16] — Estimations asymptotiques du noyau de la chaleur sur les groupes de, C. R. Math. Acad. Sci. Paris 344 (2007), no. 8, 497-502.

[17] __ Estimations optimales du noyau de la chaleur sur les groupes de type Heisenberg, J. Reine Angew. Math. 646 (2010), 195-233.

[18] - Fonctions maximales centrées de Hardy-Littlewood sur les groupes de Heisenberg, Studia Math. 191 (2009), no. 1, 89-100.

[19] R. Monti, Some properties of Carnot-Carathéodory balls in the Heisenberg group, Atti Accad. Naz. Lincei Cl. Sci. Fis. Mat. Natur. Rend. Lincei (9) Mat. Appl. 11 (2000), no. $3,155-167$.

[20] R. Monti and F. Serra Cassano, Surface measures in Carnot-Carathéodory spaces, Calc. Var. Partial Differential Equations 13 (2001), no. 3, 339-376.

[21] P. Niu, H. Zhang, and Y. Wang, Hardy type and Rellich type inequalities on the Heisenberg group, Proc. Amer. Math. Soc. 129 (2001), no. 12, 3623-3630.

[22] L. Zhu, A Hardy inequality for the Grushin type operators, Math. Inequal Appl. 12 (2012), no. 4, 923-930

School of Mathematics and Statistics

Hubei EngineERING University

Xiaogan, Hubei, P.C. 432000, P. R. China

E-mail address: $x y x 21 \mathrm{cn} @ 163 . \mathrm{com}$ 